\title{
Medrobotics Flex System for Laryngeal Surgery: A Feasible Study in Two Cadavers
}

\author{
${ }^{1}$ Soham Roy, ${ }^{2}$ Syed HS Naqvi, ${ }^{3}$ Ron J Karni
}

\section{ABSTRACT}

Objective: To report the feasibility of Medrobotics Flex ${ }^{\circledR}$ System for laryngeal surgery.

Materials and methods: Cadaver study in research laboratory.

Results: The Medrobotics Flex System was utilized with the Medrobotics Flex Retractor to evaluate robotic laryngeal surgery in a cadaver model. Two surgeons using two cadavers tested the robotic system to expose the larynx and facilitate robotic airway surgery using the Flex Instruments. In both cadavers, these procedures were performed transorally with excellent visualization: supraglottoplasty, arytenoidectomy, epiglottectomy, vocal cord lesion excision, posterior cordotomy, hemilaryngectomy, and endoscopic anterior/posterior cricoid split. Laryngeal cleft repair was limited by availability of suture to close the interarytenoid band. Laryngeal exposure was rated as "excellent" by both surgeons using the robotic platform.

Conclusion: Robotic surgery for the head and neck, while still in relative infancy, remains one of the newest platforms for the otolaryngologist. Currently available robotic platforms, while adapted to head and neck and airway surgery, were not designed for this purpose and pose challenges in size and accessibility to the larynx. The Flex System is specifically designed for head and neck and laryngeal surgery. This system provides outstanding visualization of hard to access areas in the larynx and hypopharynx. The flexible surgical instruments used with the Flex System allow for reasonable surgical exposure and technique. The Flex System may provide an improved robotic experience for transoral laryngeal and head and neck surgery.

Keywords: Laryngeal surgery, Medrobotics flex ${ }^{\circledR}$ system, Transoral.

How to cite this article: Roy S, Naqvi SHS, Karni RJ. Medrobotics Flex System for Laryngeal Surgery: A Feasible Study in Two Cadavers. Int J Head Neck Surg 2016;7(4):204-206.

Source of support: Medrobotics provided the Flex System for evaluation and cadavers for testing, along with a facility to perform the study.

\section{Conflict of interest: None}

${ }^{1}$ Vice Chairman and Associate Professor, ${ }^{2}$ Fellow, ${ }^{3}$ Chief and Associate Professor

${ }^{1-3}$ Department of Otorhinolaryngology - Head and Neck Surgery The University of Texas Health Science Center, Houston, Texas USA

Corresponding Author: Ron J Karni, Chief and Associate Professor, Department of Otorhinolaryngology - Head and Neck Surgery, The University of Texas Health Science Center, Houston, Texas, USA, Phone: +17135005410 , e-mail: ron.j.karni@uth.tmc.edu

\section{INTRODUCTION}

Minimally invasive head and neck surgery is often challenging due to difficulty in accessing the target region. Therefore, greater emphasis is being placed on transoral robotic surgery (TORS), which has emerged as a viable alternative for head and neck surgeons. The currently preferred robotic platform is the da Vinci surgical system (Intuitive Surgical Inc., Sunnyvale, CA, USA), which, while adapted to head and neck and airway surgery, was not designed for this purpose. Although TORS is frequently considered a superior option over traditional open surgery, concerns still remain with regard to the level of access and visualization of the larynx which is compromised due to the size and limited flexibility of the instruments.

A new minimally invasive technology, the Medrobotics Flex ${ }^{\circledR}$ System, has shown promising results for treating oropharynx, hypopharynx, and laryngeal disease. ${ }^{1-3}$ This robotic system is unique because of the minimal size and enhanced flexibility of both its endoscope and instruments allowing for appropriate exposure of the larynx. Currently, the Flex ${ }^{\circledR}$ Robotic System is available in Europe and has recently received Food and Drug Administration approval in the United States. ${ }^{4}$ This study seeks to investigate the feasibility of this system for laryngeal surgery in human cadaver models.

\section{MATERIALS AND METHODS}

Two senior surgeons, a head and neck oncologic surgeon (RK) and a pediatric otolaryngologist (SR), were given access to two cadavers and the Flex System to explore the options available for TORS. Both surgeons had minimal training on the system prior to attempting procedures. Surgeons were given the opportunity to try any oropharyngeal surgery or transoral laryngeal surgery using the Flex System in the adult cadavers. Both surgeons interchangeably used both cadavers and collaborated on procedures (Fig. 1).

In the course of 1 day, the following procedures were attempted by the surgeons: tonsillectomy, extended tonsil resection with parapharyngeal space dissection, uvulopalatopharyngoplasty, posterior pharyngeal flap surgery, base of tongue resection, epiglottectomy, arytenoidectomy, supraglottoplasty, posterior cordectomy, removal of vocal cord lesion, laryngeal cleft repair, endoscopic hemilaryngectomy, and endoscopic cricoid split. 


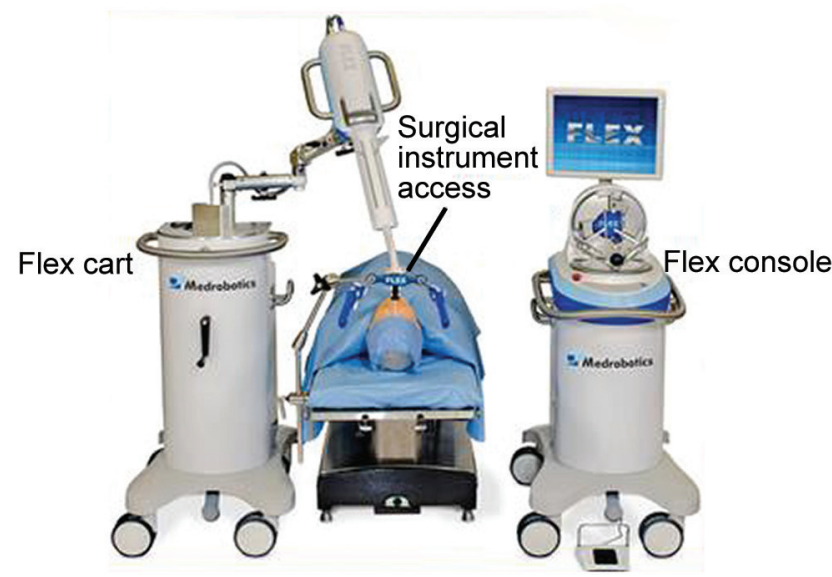

Fig. 1: Medrobotics Flex System Operating Room Layout

\section{RESULTS}

All of the attempted procedures were successful by the individual surgeons except for laryngeal cleft operation, which was not completed secondary to the availability of appropriate suture material for closure. In all cases, both surgeons rated laryngeal exposure as subjectively excellent (Fig. 2). Limitations included some difficulty with accessing areas of the anterior larynx on one cadaver, with some restriction on exposure. Additionally, some limitation in the instrumentation with the Flex System resulted in breakage of grasping instruments. Both surgeons in postreview comments reported excellent exposure and good instrumentation for oropharyngeal and hypopharyngeal surgery, with excellent visualization and some limitations of instrumentation for laryngeal surgery.

\section{DISCUSSION}

Transoral robotic surgery is now considered an advantageous technology for minimally invasive endoscopic surgical treatment of head and neck disorders. Among the current technologies, the sophisticated da Vinci surgical system (Intuitive Surgical, City State) is the most recognized. The da Vinci surgical system has four

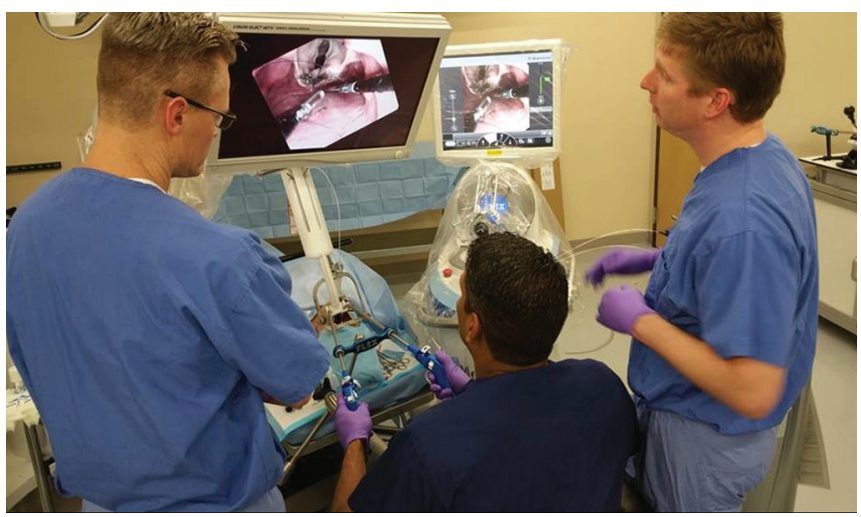

Fig. 2: Surgeon using the Flex System surgical arms, which are equipped with wristed-tip instruments and have the capability to turn and rotate, allowing for improved maneuverability in compact areas. ${ }^{5}$ Furthermore, it has a magnified three-dimensional high-definition camera with the ability to zoom in or out, providing the surgeon with quality imaging. These advanced functions have allowed this platform to become a global leader in terms of minimally invasive robotic surgery, including head and neck surgery.

Certainly, there are advantages of applying the da Vinci surgical system for various minimally invasive procedures. However, this system was not originally designed for head and neck surgery. The size of the instrument manipulators ( $5 \mathrm{~mm}$ diameter) has been a major limiting factor for the use of this system in ENT surgery, particularly of the larynx and hypopharynx. The anatomy of the upper aerodigestive tract limits maneuverability of the robotic arms and requires another physician's hand to separate them. ${ }^{6}$ This results in less space for additional instruments like suctions or oral retractors, further deteriorating the ability to move freely in an already narrow anatomical location. In addition, instruments including the 3D camera (12 $\mathrm{mm}$ diameter) on the da Vinci robot are rigid and add greater difficulty to operate in constricted areas. McLeod et $\mathrm{al}^{7}$ reported that this dilemma has led to occasional internal binding and abutting at the operative area. We have previously reported on robotic laryngeal surgery in the laryngoscope.

Recently in the United States, interest in the application of an innovative, minimally invasive, transoral robotic platform known as the Medrobotics Flex ${ }^{\circledR}$ System has emerged. This novel platform is unique in that it was designed intentionally for patients undergoing transoral head and neck surgical procedures. The Flex ${ }^{\circledR}$ System has highly flexible "snake-like" endoscopic tools that provide surgeons an improved likelihood of complete visualization and access for tissue manipulation in laryngeal surgery (Fig. 3). Friedrich et $\mathrm{al}^{8}$ described that

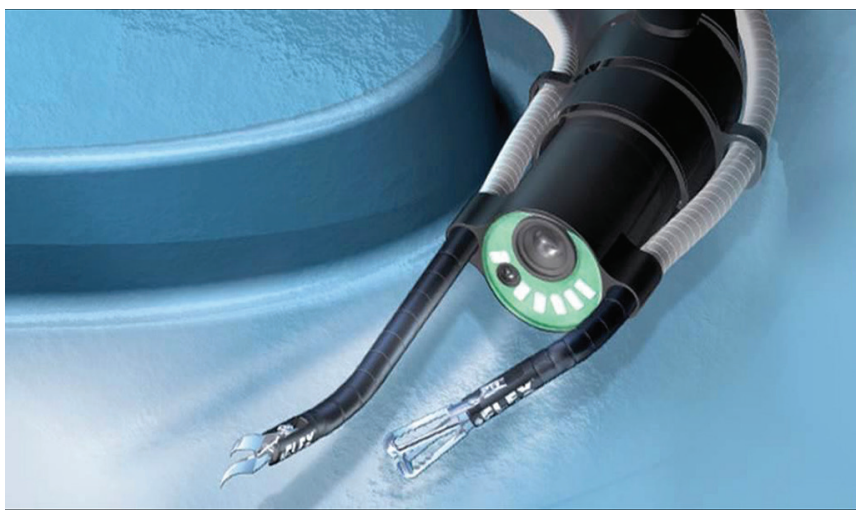

Fig. 3: Medrobotics Flex System Robotic Arm 
the flexibility of the instruments on the Flex ${ }^{\circledR}$ System allowed for improved surgical access and nontraumatic approach in patients with challenging anatomy. The robot has a two-dimensional high-definition digital camera located on the distal end, which relays images via a specialized "chip-on-a-tip"-type scope. ${ }^{1}$ However, a three-dimensional camera will likely be available in the near future.

The experience of these authors suggests that this flexible camera system provides excellent exposure and access to previously difficult-to-reach areas in the hypopharynx and larynx via traditional robotic technologies. Indeed, this study suggests that the Medrobotics Flex ${ }^{\circledR}$ System may potentially improve surgery of laryngeal and hypopharyngeal diseases, which were previously difficult to reach with the da Vinci system. However, important variables that require further consideration include operation time, functional outcome, cost, safety, and efficacy. While our findings were based on cadaveric experience, multiinstitutional studies should be encouraged in order to definitively assess the feasibility of this platform in real-time laryngeal surgery.

\section{CONCLUSION}

The Medrobotics Flex ${ }^{\circledR}$ System, when applied on a cadaveric model, provided exceptional visualization of hard to access areas of both the larynx and hypopharynx when compared with traditional robotic platforms. Moreover, the stability and flexibility of the instruments of the Flex ${ }^{\circledR}$ System allowed for excellent exposure and technique.
Hence, we believe the Flex ${ }^{\circledR}$ System may potentially provide an improved robotic experience for surgeons performing transoral head and neck surgery, especially of the larynx.

\section{REFERENCES}

1. Johnson PJ, Rivera Serrano CM, Castro M, Kuenzler R, Choset H, Tully S, Duvvuri U. Demonstration of transoral surgery in cadaveric specimens with the Medrobotics flex system. Laryngoscope 2013 May;123(5):1168-1172.

2. Mandapathil M, Duvvuri U, Guldner C, Teymoortash A, Lawson G, Werner JA. Transoral surgery for oropharyngeal tumors using the Medrobotics(®)Flex(®) System - a case report. Int J Surg Case Rep 2015 Mar;10:173-175.

3. Hasskamp P, Lang S, Holtmann L, Stuck BA, Mattheis S. First use of a new retractor in transoral robotic surgery (TORS). Eur Arch Otorhinolaryngol 2015 Jul;273(7):1913-1917.

4. Mendelsohn A, Lawson G, Remacle M. Transoral robotic surgery of the larynx and airway. Robotic Surg Head Neck 2015 Jan;83-97.

5. Hillel AT, Kapoor A, Simaan N, Taylor RH. Applications of robotics for laryngeal surgery. Otolaryngol Clin North Am 2013;41(4):781-791.

6. Esteban F, Menoyo A, Abrante A. Critical analysis of robotic surgery for laryngeal tumours. Acta Otorrinolaringol Esp 2014 Nov-Dec;65(6):365-372.

7. McLeod IK, Mair EA, Melder PC. Potential applications of the da Vinci minimally invasive surgical robotic system in otolaryngology. Ear Nose Throat J 2005 Aug;84(8):483-487.

8. Friedrich DT, Scheithauer MO, Greve J, Duvvuri U, Sommer F, Hoffmann TK, Schuler PJ. Potential advantages of a singleport, operator-controlled flexible endoscope system for transoral surgery of the larynx. Ann Otol Rhinol Laryngol 2015 Aug;124(8):655-662. 